\title{
ENVIRONMENTAL AND GLOVES' CONTAMINATION BY STA- PHYLOCOCCI IN DENTAL HEALTHCARE SETTINGS
}

\author{
Giuseppe A. M essano ${ }^{1}$, Virgilio De B ono ${ }^{2}$, Renato Architrave ${ }^{1}$, Stefano Petti ${ }^{1}$ \\ ${ }^{1}$ SAPIENZA UNIVERSITY, DEPARTMENT OF PUBLIC HEALTH AND INFECTIOUS DISEASES, ROME, ITALY; \\ 2ITALIAN MEDICAL ASSOCIATION, ROMAN SECTION, ROME, ITALY
}

\section{Abstract}

Introduction. Each year, 37,000 people in Europe die as direct consequence of healthcare-associated infections. Staphylococcus aureus (especially methicillin-resistant-MRSA) and coagulase-negative staphylococci (CNS) are frequently isolated in these episodes. Environmental contamination by $S$. aureus, MRSA and CNS in dental healthcare settings is reported moderately frequently, although the associated risk for infection is not clear.

Aim. To investigate contamination of disposal gloves and of clinical contact surfaces by several types of staphylococci in dental offices soon after dental therapy.

Material and methods. 136 general dental practitioners (GDPs) voluntarily participated. At each sampling occasion, environmental samples were collected from the tray and from the gloved dominant hand, soon after dental therapy of the second or third patient of the working session. Contact plates containing Mannitol Salt Agar were used. Overall staphylococci, S. aureus, CNS and Staphylococcus epidermidis (member of CNS group) were presumptively identified and resistance to oxacillin was tested to identify methicillin-resistant (MR) strains.

Results. Staphylococci were detected in $41 \%$ and $57 \%$ samples from trays and from gloves, respectively; S. aureus in 5\% and $5 \%$, CNS in $36 \%$ and $52 \%$, S. epidermidis in $18 \%$ and $44 \%$, methicillin-resistant S. aureus (MRSA) in 1.5\% and $1.5 \%$, MRCNS in $1.5 \%$ and $2.2 \%, M R-S$. epidermidis in $1.5 \%$ and $1.5 \%$. The samples collected from the trays were correlated with those collected from hands for all these types of staphylococci.

Conclusion. Although it was not possible to ascertain the main source of staphylococci contamination -patient or GDP, dominant hands and clinical contact surfaces were frequently contaminated.

Key words: dentistry, clinical contact surface, gloves, general dental practitioner, Staphylococcus aureus, coagulase-negative staphylococci, Staphylococcus epidermidis

\section{Introduction}

Healthcare-associated infections (HAI) occur after exposure to healthcare. Each year, 4,000,000 people in Europe acquire HAIs and 37,000 of them die as the direct consequence of infection. In addition, HAIs may also interest healthcare workers and administrative staff. The most frequent HAI types are urinary tract infections, pneumonia, surgical site infections, bloodstream infections and gastrointestinal infections, while the most frequently isolated microorganisms in HAI overall are Escherichia coli, Staphylococcus aureus, Pseudomonas aeruginosa, Enterococcus species, coagulase-negative staphylococci and Candida species ${ }^{1}$. HAIs are generally treated through antibiotic and/or surgical therapy. Therefore, HAI treatment and prevention is an important cause of emergence of highly antibiotic resistant strains, another serious public health problem ${ }^{2}$.

In the specific field of dental healthcare settings, environmental contamination is reported for methicillin-resistant and methicillin-sensitive S. aureus (MRSA and MSSA), although the risk for infection associated with contamination is not clear ${ }^{3-5}$. One problem with MRSA in dentistry is that these micro-organisms may survive up to six months on clinical contact surfaces in the dental environment ${ }^{6}$. P. aeruginosa and Legionella pneumophila, microorganisms responsible for HAIs, also are frequently de-

(C) 2013 Faculty of Medicine in Niš. Clinic of Dentistry in Niš. All rights reserved / (C) 2013 Medicinski fakultet Niš. Klinika za stomatologiju Niš. Sva prava zadržana
Address for correspondence:

Dr. Giuseppe AlessioMessano

Department of Public Health and Infectious Diseases

Sapienza University

P.le Aldo Moro 5, 00185 Rome, Italy

Phone/Fax: +3906 49914667

Email: giuseppe.messano@yahoo.it 
tected in dental unit waterlines, along with oral streptococci, biological markers of bloodborne/ airborne pathogens ${ }^{7-9}$. The study of environmental contamination and consequent infection transmission in dental healthcare settings is, therefore, essential to produce evidence-based guidelines ${ }^{10-12}$.

\section{Aim}

The aim of the present study was to investigate the contamination level of the environment of dental offices and of the hands of the dental staff by several types of staphylococci.

\section{Material and M ethods}

One hundred thirty-six General Dental Practitioners (GDPs) working in private offices in Rome, Italy, were considered. Details regarding their recruitment were previously described ${ }^{13}$; GDPs were invited to participate before registering to Continuing M edical Education courses. Participation was on a voluntary basis and there were no incentives. Data protection and anonymity were guaranteed. The study protocol was approved by the Review Board of the Medical and Dental Association of Rome.

Samples were collected in the midmorning/ midafternoon. GDPs must have treated at least two patients before the sampling occasion. Environmental samples were collected from the tray in front of the patient, a clinical contact surface ${ }^{10}$, immediately after patient treatment and before surface cleaning, using disposable swabs previously imbibed in sterile water and passed on a 10x10 cm area of the tray. ATP bioluminescence (Lumicontrol II. PBI International, Milan, Italy) was used to assess the overall contamination level, qualitatively. ATP bioluminescence is a rapid method to detect the total viable flora, with a reasonably good accuracy of $114 \%$ at levels around 100 Relative Light Units (RLUs), roughly corresponding to 2.5 colony forming units (CFU)/cm2. 100 RLUs is generally considered the benchmark for high level of environmental cleanliness in hospitals 14,15 .

Samples which yielded RLU levels $>100$ were monitored using Replicate Organism Detection and Counting (Rodac) plates (Becton Dickinson Italia, Buccinasco, Italy) containing
Mannitol Salt Agar (MSA - Becton Dickinson Italia), for the enumeration of staphylococci. Plates were pressed on the surface of the tray different from the area sampled with the swab for $30 \mathrm{~s}$ at an approximate pressure of 20-25 g/ $\mathrm{cm}^{2}$. Rodac plates were preferred to swab because they provide repeatable results and provide comparable levels of recovery for general bacterial contamination of a surface ${ }^{16}$ Plates were aerobically incubated at $37^{\circ} \mathrm{C}$ for $48 \mathrm{~h}$. Colonies grown on M SA were Gram's stained, tested for coagulase and catalase and presumptively identified using VITEK-2 "Gram-Positive Identification" and "Antibiotic Susceptibility Testing" cards (BioMérieux, Italia; Bagno a Ripoli, Italy). Further biochemical identification tests were not made, therefore, micro-organisms were presumptively classified as overall staphylococci, S. aureus, coagulase negative staphylococci (CNS) and S. epidermidis, a subgroup of CNS.

Following the criteria of the US Centers for Disease Control, isolated staphylococci strains were classified as methicillin-resistant on the basis of oxacillin MIC test, which implied the presence of the staphylococcal cassette chromosome mec (SCCmec). Namely, $\geq 4 \mu \mathrm{g} / \mathrm{mL}$ for $\mathrm{S}$. aureus and $\geq 0.5 \mu \mathrm{g} / \mathrm{mL}$ for CNS (guidelines available at, http://www.cdc.gov/mrsa/ lab/lab-detection.html).

Right hand samples among right-handed GDPs (from left hand among left-handed GDPs) were collected directly from the gloved hand of GDPs soon after patient treatment and before glove removal using four Rodac plates containing the aforementioned media and following the same laboratory procedures used for the environmental samples.

Staphylococci prevalence estimates on clinical contact surfaces and on GDPs' gloved dominant hands were assessed. The association between clinical contact surface and gloved hand contamination by the various Staphylococcus species also was assessed using the nonparametric Spearman correlation test, giving score 1 to positive samples and score 0 to negative samples.

GDPs were informed in the event that samples were positive for methicillin-resistant staphylococci. Environmental samples and samples from GDPs' nares were collected using sterile swabs one week after the sampling occasion to check whether additional procedures 
were necessary to eradicate these microorganisms.

\section{Results}

Seventy-six (55.9\%) clinical contact surfaces provided RLU levels higher than 100. Overall presumptive staphylococci prevalence was $41.2 \%(\mathrm{n}=56,73.7 \%$ of all ATP bioluminescence positive samples), with $5.1 \% \mathrm{~S}$. aureus $(n=7)$ and $36.0 \%(n=49)$ CNS, of whom $18.4 \%$ $(\mathrm{n}=25)$ S. epidermidis (Table 1). Two presumptive $\mathrm{S}$. aureus species, corresponding to $1.5 \%$
S. epidermidis $(1.5 \%)$ were methicillin resistant (Table 2).

A good correlation was found between positive environmental samples and positive samples from gloves for overall staphylococci, S. aureus and CNS (Table 1; $<<0.0001$ ). As for methicillin-resistant staphylococci, all the MRSA $(n=2)$ were isolated from the environmental sample and on GDP's glove at the same time, while one of four CNS (S. epidermidis) was isolated from both samples (Table 2). In the three sampling occasions where methicillinresistant staphylococci were isolated from the tray and from the glove of the dominant hand at

Table 1. Prevalence (95\% confidence interval -95CI- between square brackets) of samples with ATP bioluminescence levels $>100$ Relative Light Units (RLU -corresponding to $2.5 \mathrm{CFU} / \mathrm{cm} 2$ ) and of the investigated staphylococci detected immediately after dental therapy on the 136 clinical contact surfaces and GDPs' gloved hands. Association between staphylococci detected on clinical contact surfaces and staphylococci detected on GDP's gloved hands (Spearman correlation coefficient $\rho$-rho- corrected for ties).

\begin{tabular}{llllll}
\hline & $\begin{array}{l}\text { R } 100 \\
\end{array}$ & staphylococci & S. aureus & CNS & S. epidermidis \\
\hline clinical contact surfaces & $55.9 \%(\mathrm{n}=76)$ & $41.2 \%(\mathrm{n}=56)$ & $5.1 \%(\mathrm{n}=7)$ & $36.0 \%(\mathrm{n}=49)$ & $18.4 \%(\mathrm{n}=25)$ \\
& {$[47.6-64.2]$} & {$[32.9-49.5]$} & {$[1.4-8.8]$} & {$[27.9-44.1]$} & {$[11.9-24.9]$} \\
gloved hands & & $57.4 \%(\mathrm{n}=78)$ & $5.1 \%(\mathrm{n}=7)$ & $52.2 \%(\mathrm{n}=71)$ & $44.1 \%(\mathrm{n}=60)$ \\
Spearman $\rho$ & & {$[49.1-65.7]$} & {$[1.4-8.8]$} & {$[43.8-60.6]$} & {$[35.8-52.4]$} \\
& & 0.66 & 0.70 & 0.72 & 0.31 \\
& $(\mathrm{p}<0.0001)$ & $(\mathrm{p}<0.0001)$ & $(\mathrm{p}<0.0001)$ & $(\mathrm{p}=0.0004)$ \\
\hline
\end{tabular}

CNS: coagulase negative staphylococci (the species S. epidermidis is a member of CNS)

of all samples $(3.6 \%$ of the environmental detected staphylococci, $28.6 \%$ of $\mathrm{S}$. aureus isolates), resulted methicillin resistant. Two S. epidermidis isolates (1.5\% of all samples, $3.6 \%$ of staphylococci isolates) were methicillin resistant (Table 2). the same time, the two microorganisms exhibited the same antibiotic profile, suggesting that the strain detected on the tray was the same as the strain detected from the glove (data not in Table).

Table 2. Prevalence (95\% confidence interval -95CI- between square brackets) of methicillin-resistant staphylococci isolated from the 136 clinical contact surfaces and the GDPs' gloved hands.

\begin{tabular}{lllll}
\hline & staphylococci & S. aureus & CNS & S. epidermidis \\
\hline clinical contact surfaces & $2.9 \%(\mathrm{n}=4)$ & $1.5 \%(\mathrm{n}=2)$ & $1.5 \%(\mathrm{n}=2)$ & $1.5 \%(\mathrm{n}=2)$ \\
& {$[<0.1-5.7]$} & {$[<0.0-3.5]$} & {$[<0.0-3.5]$} & {$[<0.0-3.5]$} \\
gloved hands & $3.7 \%(\mathrm{n}=5)$ & $1.5 \%(\mathrm{n}=2)$ & $2.2 \%(\mathrm{n}=3)$ & $1.5 \%(\mathrm{n}=2)$ \\
& {$[0.5-6.9]$} & {$[<0.0-3.5]$} & {$[<0.0-4.7]$} & {$[<0.0-3.5]$} \\
\hline
\end{tabular}

The two methicillin-resistant S. aureus isolates and two out of 3 methicillin-resistant CNS isolates (one S. epidermidis) were isolated on the clinical contact surface and on the GDP's gloved hand at the same sampling occasions.

Overall staphylococci prevalence in samples from gloves soon after dental therapy was $57.4 \%(\mathrm{n}=78)$, S. aureus accounted for $5.1 \%$ $(\mathrm{n}=7)$ of the samples while the remaining $52.2 \%$ $(n=71)$ were CNS, including $44.1 \%(n=60) \mathrm{S}$. epidermidis (Table 1). Two presumptive S. aureus species, corresponding to $1.5 \%$ of samples (2.6\% of the detected staphylococci, $28.6 \%$ of all S. aureus isolates), were methicillin resistant. Three CNS (2.2\%) and, among them, two
In the six dental offices where methicillinresistant staphylococci were detected, environmental samples, collected one week following the first sampling occasion, provided negative results regarding the occurrence of methicillinresistant staphylococci. Samples from GDPs' nares also resulted negative. 


\section{Discussion}

The present study is one of the papers presented at the workshop "Advances in Infection Epidemiology and Control in Dental Healthcare Settings", Department of Public Health and Infectious Diseases, Sapienza University, Rome, Italy on February 9th, 2013 17-23.

MRSA infection transmission is a serious public health problem and a cause for concern among dental healthcare workers and patients ${ }^{24}$. While MRSA infection can be transmitted to patients and to healthcare workers particularly in certain settings, such as intensive care or surgical units ${ }^{25}$, there are no ascertained cases of transmission to dental healthcare workers and MRSA carriage rates among GDPs are generally similar to or even lower than the general population, thus suggesting that the occupational risk of MRSA infection is probably mini$\mathrm{mal}^{3}$. The question regarding the risk of acquiring MRSA infection among dental patients is more complex. Among special patients, such as special care patients, hospitalized patients, head and neck cancer patients and oral or maxillofacial surgery patients, high MRSA carriage rates are reported, as well as frequent episodes of infection and colonization, other than environmental contamination of clinics and units. These elements suggest that the risk of infection is high among these patients. The situation among the remaining dental patients is different, as there are only two reported episodes of MRSA infection, one of them due to the lack of glove use by the dentist. Carriage rate among healthy adult dental patients also is low, while there was only one case of M RSA environmental detection in non-special and non-surgical dental departments or offices ${ }^{3,4}$. These elements collectively suggest that the risk for infection is generally low.

The present study showed that MRSA spread in dental healthcare settings is possible. Indeed, MRSA were detected in two out of the 136 sampling occasions collected soon after dental therapy and in these occasions both trays and gloves from the dominant hand resulted contaminated by these microorganisms at the same time. Although no further analysis was made to ascertain whether the strains detected on the gloves and those detected on the trays were the same, it is likely that it was so, because the strain detected from the glove exhibited the same antibiotic profile as the strain detected from the tray. This assumption, along with the reported high correlation between staphylococci, CNS, S. aureus and S. epidermidis detected in the environment and those detected on the gloves may suggest two possible hypotheses to explain MRSA spread in the dental offices and the consequent risk for infection. First hypothesis: GDPs were MRSA carriers and picked these microorganisms from their skin/mucosae while they were wearing gloves, spreading them in the environment. Second hypothesis: the dental patient under treatment was MRSA carrier and was touched by GDP's gloved dominant hands, which in turn, contaminated the tray in front of the patient or, alternatively, airborne MRSA from carrier patient contaminated the tray. In favour of the second hypothesis the fact that GDPs resulted free from MRSA one week after MRSA detection in their offices, therefore, these two GDPs could only be transient carriers. Dispersion of airborne staphylococci during dental therapy also is corroborated by studies reporting CNS prevalence of $10-35 \%$ in air samples from dental clinics and offices ${ }^{26-28}$ a similar detection rate as that reported in the present study (Table 1).

\section{Conclusion}

In conclusion, environmental contamination by methicillin-resistant and methicillinsensitive staphylococci during dental therapy is possible and may pose a risk for infection, particularly among immune-depressed patients and those with open lesions in mouth. However, it seems likely that routine glove change between patients and cleaning/disinfection of clinical contact surfaces could be sufficient to control such a risk for infection, but to be sure that these simple methods are applied, it is necessary that the overall level of knowledge and awareness among dental healthcare workers is improved, perhaps through specific Continuing M edical Education courses, as issues regarding infection control are generally neglected among dental healthcare workers ${ }^{29}$. 


\section{LITERATURA / REFERENCES}

1. European Centre for Disease Prevention and Control. Surveillance of healthcare-associated infections in Europe, 2007. Stockholm: ECDC; 2012.

2. Pittet D, Allegranzi B, Sax H et al. Considerations for a WHO European strategy on health-care-associated infection, surveillance, and control. Lancet Infect Dis 2005;5(4):242-50.

3. Petti S, Polimeni A. Risk of methicillin-resistant Staphylococcus aureus transmission in the dental healthcare setting: a narrative review. Infect Control Hosp Epidemiol 2011;32(11):1109-15.

4. Roberts MC, Soge OO, Horst JA, Ly KA, Milgrom P. Methicillin-resistant Staphylococcus aureus from dental school clinic surfaces and students. Am J Infect Control 2011;39(8):628-32.

5. Petti S, Polimeni A. Methicillin-resistant Staphylococcus aureus infection transmission in dental health care settings: myths and facts. Am J Infect Control 2012;40(3):287-8.

6. Petti S, De Giusti M, Moroni C, Polimeni A. Long -term survival curve of methicillin-resistant Staphylococcus aureus on clinical contact surfaces in natural-like conditions. Am J Infect Control 2012;40(10):1010-2.

7. Walker JT, Bradshaw DJ, Finney $M$ et al. Microbiological evaluation of dental unit water systems in general dental practice in Europe. Eur J Oral Sci 2004;112(5):412-8.

8. O’Donnell MJ, Boyle MA, Russell RJ, Coleman DC. Management of dental unit waterline biofilms in the 21st century. Future Microbiol 2011;6(10):1209-26.

9. Petti S, Moroni C, Messano GA, Polimeni A. Detection of oral streptococci in dental unit water lines after therapy with air turbine handpiece: biological fluid retraction more frequent than expected. Future Microbiol 2013;8(3):413-21.

10. K ohn W G, Collins A S, Cleveland JL et al. Guidelines for infection control in dental health-care settings, 2003. MMWR Recomm Rep 2003;52(RR-17):1-61.

11. Petti S, Polimeni A. The rationale of guidelines for infection control in dentistry: precautionary principle or acceptable risk? Infect Control Hosp Epidemiol 2010;31(12):1308-10.

12. Laheij AM, Kistler JO, Belibasakis GN, Välimaa $\mathrm{H}$, de Soet JJ; European Oral Microbiology Workshop (EOMW) 2011. Healthcare-associated viral and bacterial infections in dentistry. J Oral Microbiol 2012. doi: 10.3402/jom.v4i0.17659.

13. Messano GA, Petti S. General dental practitioners and hearing impairment. J Dent 2012;40(10):821-8.

14. Lewis T, Griffith C, Gallo M, Weinbren M. A modified ATP benchmark for evaluating the cleaning of some hospital environmental surfaces. J Hosp Infect 2008;69(2):156-63.

15. Mulvey D, Redding P, Robertson C, Woodall C, Kingsmore P, Bedwell D, Dancer SJ. Finding a benchmark for monitoring hospital cleanliness. J Hosp Infect 2011;77(1):25-30.
16. Galvin S, Dolan A, Cahill O, Daniels S, Humphreys H. Microbial monitoring of the hospital environment: why and how? J Hosp Infect 2012;82(1):14351.

17. Messano GA, Sofan AAA, Petti S. Quality of air and water in dental healthcare settings during professional toothcleaning. A cta Stomatol Naissi 2013; 29(67): 1230-35; doi: 10.5937/asn1367230M.

18. Messano GA, Masood M, Palermo P, Petti S. Predictors of Legionella occurrence in dental unit waterlines of a highly colonized dental hospital. Acta Stomatol Naissi 2013; 29(67): 1236-41; doi: 10.5937/asn1367236M.

19. Messano GA, Masood M, Palermo P, Petti S. Prevalence of reactive tuberculin skin test in dental healthcare workers and students. Acta Stomatol Naissi 2013; 29(67): 1242-48; doi: 10.5937/asn1367242M.

20. Petti S, Sofan AAA, Messano GA. Streptococcus pneumoniae carriage rate in healthy preadolescent dental patients. Acta Stomatol Naissi 2013; 29(67): 1249-54; doi: 10.5937/asn1367249P.

21. Messano GA. Bacterial and fungal contamination of dental hygienists' hands with and without finger rings. A cta Stomatol Naissi 2013; 29(67): 1260-64; doi: 10.5937/asn1367260M .

22. Petti S, Messano GA, Polimeni A, Dancer SJ. Effect of cleaning and disinfection on naturally contaminated clinical contact surfaces. A cta Stomatol Naissi 2013; 29(67): 1265-72; doi: 10.5937/asn1367265P.

23. Petti S, Messano GA, Scully C. Antibody level and immunity against Hepatitis B virus infection among general dental practitioners. Acta Stomatol Naissi 2013; 29(67): 1273-78; doi: 10.5937/asn1367273P.

24. Klevens RM, Gorwitz RJ, Collins AS. Methicillin-resistant Staphylococcus aureus: a primer for dentists. J Am Dent Assoc 2008;139(10):1328-37.

25. Albrich WC, Harbarth S. Health-care workers: source, vector, or victim of MRSA? Lancet Infect Dis 2008;8(5):289-301.

26. Rautemaa R, Nordberg A, Wuolijoki-Saaristo $\mathrm{K}$, Meurman JH. Bacterial aerosols in dental practic -a potential hospital infection problem? J Hosp Infect 2006;64(1):76-81.

27. Szymańska J, Dutkiewicz J. Concentration and species composition of aerobic and facultatively anaerobic bacteria released to the air of a dental operation area before and after disinfection of dental unit waterlines. Ann Agric Environ Med 2008;15(2):301-7.

28. Kimmerle H, Wiedmann-Al-Ahmad M, Pelz K, Wittmer A, Hellwig E, Al-A hmad A. A irborne microbes in different dental environments in comparison to a public area. Arch Oral Biol 2012;57(6):689-96.

29. Petti S, Messano GA, Polimeni A. Dentists' awareness toward vaccine preventable diseases. Vaccine. 2011;29(45):8108:12. 